\title{
Dorsal hand vein authentication system using artificial neural network
}

\author{
Sze Wei Chin ${ }^{1}$, Kim Gaik Tay ${ }^{2}$, Chang Choon $\mathrm{Chew}^{3}$, Audrey Huong ${ }^{4}$, Ruzairi Abdul Rahim ${ }^{5}$ \\ 1,2,3,4 Department of Electronic Engineering, UTHM, Malaysia \\ ${ }^{5}$ Department of Control and Instrumentation Engineering, UTM, Malaysia
}

\begin{tabular}{|c|c|}
\hline Article Info & ABSTRACT \\
\hline Article history: & \multirow{10}{*}{$\begin{array}{l}\text { Biometric feature authentication technology had been developed and } \\
\text { implemented for the security access system. However, the known biometric } \\
\text { features such as fingerprint, face and iris pattern failed to provide ideal } \\
\text { security. Dorsal hand vein is the features beneath the skin which makes it not } \\
\text { easily be duplicated and forged. It was expected to be used in biometric } \\
\text { authentication technology to achieve an ideal accuracy with the uniqueness } \\
\text { of its characteristics. In this study, } 240 \text { images of } 80 \text { users were obtained } \\
\text { from Bosphorus Hand Vein Database. The images were then pre-processed } \\
\text { by cropping ROI, mean filtering, CLAHE enhancing and histogram } \\
\text { equalizing. The ROI was then segmented by implementing binarization. The } \\
\text { local binary pattern (LBP) features were then extracted from the binarized } \\
\text { image. The extracted features were sent to an artificial neural network } \\
\text { (ANN) for the classification of the images. The training result shows that the } \\
\text { LBP features and ANN can recognize the dorsal hand vein pattern quite well } \\
\text { with } 90 \% \text { recognition rate. The NN net was then utilized in the MATLAB } \\
\text { GUI program for testing } 100 \text { images ( } 80 \text { trained images of } 80 \text { users and } 20 \\
\text { untrained images of } 20 \text { users) from the Bosphorus Hand Vein Database. The } \\
\text { results revealed } 100 \% \text { accuracy in their matching result. }\end{array}$} \\
\hline Received Sep 25, 2020 & \\
\hline Revised Nov 27, 2020 & \\
\hline Accepted Dec 12, 2020 & \\
\hline Keywords: & \\
\hline Artifcial neural network & \\
\hline Biometric authentication & \\
\hline Classification & \\
\hline Dorsal hand vein & \\
\hline LBP features & \\
\hline
\end{tabular}

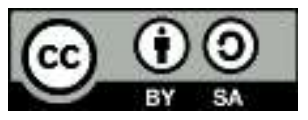

Corresponding Author:

Kim Gaik Tay

Department of Electronic Engineering

UTHM, 86400 Parit Raja,

Batu Pahat, Johor, Malaysia

Email: tay@uthm.edu.my

\section{INTRODUCTION}

Biometric feature authentication technology had been developed and implemented for the security access system. Biometric is defined as the term used in computer science to refer to the field of mathematical analysis of human features such as fingerprint, palm, finger veins, eyes, voice, signature, gait and DNA [1]. By comparing to the traditional personal verification method, such as password, PINS, magnetic swipe cards, keys and smart cards which only offer limited security and are unreliable. Biometric feature authentication technology offers a more reliable and secure performance as biometric features are hard to forge and relatively easy to use [2].

From the past studies, the biometric features such as fingerprint, iris and face that used in the current biometric recognition still fail to provide a highly secure environment. The fingerprint could be produced by a gelatin mould to fool the fingerprint reader, the brightness of the surroundings will affect the detection of iris pattern besides the different view of the face captured increases the difficulty to recognize a face [3]. 
Biometric authentication systems based on dorsal hand vein patterns had gained attention in recent years due to their physiological properties which are unique, universal and immune to the imposter attacks [4]. Dorsal hand vein is one of the vascular patterns at the back of the hand. This characteristic has made dorsal hand vein not easily to be damaged, wore, duplicated and counterfeited as it is beneath the skin [5, 6]. Apart from this, the dorsal hand vein is believed to be different for every individual, even the twins will have different vein pattern [5]. By comparing to fingerprints, iris and face, the dorsal hand vein provides higher security in the biometric recognition system.

Past studies [2, 7-12] suggested imaging sensors such as a complementary metal-oxidesemiconductor (CMOS) or charged-coupled device (CCD) camera, scanner and webcam with the aid of an infrared source or a neutral density filter. Nevertheless, some researchers preferred to use valid databases, such as a dorsal hand vein image database from [13], North China University of Technology (NCUT) hand dorsal vein database [14], general primary data source (GPDS) [15] and Bosphorus Hand Vein Database $[16,17]$.

In the image pre-processing stage, obtaining region of interest (ROI), enhancing and denoising images are essential to make the vein pattern easily to be detected at the next segmentation stage. Some researchers implemented algorithms, such as devising datum points [2], Knuckle extraction [2, 11, 18], grey normalization methods [10] to extract ROI of dorsal hand vein. Among the techniques to enhance the ROI, include histogram equalization techniques like contrast limited adaptive histogram equalization (CLAHE) [19], adaptive histogram equalization (AHE) [11, 20, 21] and equalized histogram [4]. Meanwhile, researchers applied Wiener filter, smoothing filter, median filter and 2-D Gaussian filter to reduce the noise in the image [3, 22-24].

In the effort to segment out the dorsal vein patterns from the image, a thresholding technique such as local dynamic or adaptive threshold with morphological operation [4, 9, 24, 25] was proposed. Binarization was also recommended in [16] as it segments out the ROI into white and the black part is the background. By referring to the past studies, local binary pattern (LBP) was suggested to extract the features of dorsal hand vein pattern $[3,5,10]$. LBP, a simple yet efficient texture operator labels the pixels of the images by thresholding the neighbourhood of each pixel and consider the result as a binary number.

Classification is required in a biometric recognition system to identify and recognize the class of images. Winner-takes-all-rule [6] which means that the value ' 1 ' indicates the correct class, while the value ' 0 ' indicates the incorrect class and cross-entropy error was used to detect errors. K-nearest neighbor (KNN), support vector machine (SVM), Random forest [26] and Mahalanobis distance [25] were used to classify the images by the past researches.

Even though different techniques for a dorsal hand vein recognition were proposed in the past, there is a need for a higher accuracy system. This is because, according to the previous study, the accuracy of the existing approach is limited to the range $51-98 \%$, or an error range of $2-49 \%$. Thus, this study intends to perform dorsal hand vein pattern recognition using LBP features and develop a dorsal hand vein authentication system using artificial neural network (ANN) besides evaluating the performance accuracy of the dorsal vein pattern authentication system.

\section{RESEARCH METHOD}

In this work, the dorsal hand vein pattern authentication using ANN was categorized into two phases, that were training phase and authentication phase. The workflow of this paper is displayed in Figure 1. Bosphorus Hand Vein Database which has a total of 1575 hand images from 100 users under different conditions as seen in Table 1 was employed in this study.

Table 1. Classification of images

\begin{tabular}{|c|c|c|c|c|}
\hline Number & Conditions & Left-hand & Right-hand & Number of images \\
\hline 1 & Normal (N) & 3 images per user & 3 images per user & 600 \\
\hline 2 & After carrying a $3 \mathrm{~kg}$-bag for $60 \mathrm{~s}(\mathrm{~B})$ & 3 images per user & - & 300 \\
\hline 3 & $\begin{array}{l}\text { After squeezing an elastic ball (closing and } \\
\text { opening) for } 60 \mathrm{~s}(\mathrm{~A})\end{array}$ & 3 images per user & - & 300 \\
\hline 4 & $\begin{array}{l}\text { After cooling the hand by holding the ice pack on } \\
\text { the surface of the back of the hand. (I) }\end{array}$ & 3 images per user & - & 300 \\
\hline 5 & $\begin{array}{c}\text { After a time-lapse ranging from two months to } \\
\text { five months. }\end{array}$ & - & - & 75 \\
\hline
\end{tabular}


In this project, 100 users' left-hand images under different conditions ( $(34$ users from $\mathrm{N}$-condition, 34 users from A-condition and 32 users from I- condition) were selected. The $\mathrm{N}$ - condition, A- condition and I- condition will cover hand vein conditions during tensed and relaxed conditions. Among the 100 users, 80 users were selected to act as genuine users, while the rest 20 users were used to act as the imposters. Thus, in the training phase, a total of 240 images of 80 genuine users was used. Whereas, in the authentication phase, 100 images (80 images from 80 genuine users and 20 images from 20 imposters) were used for testing.

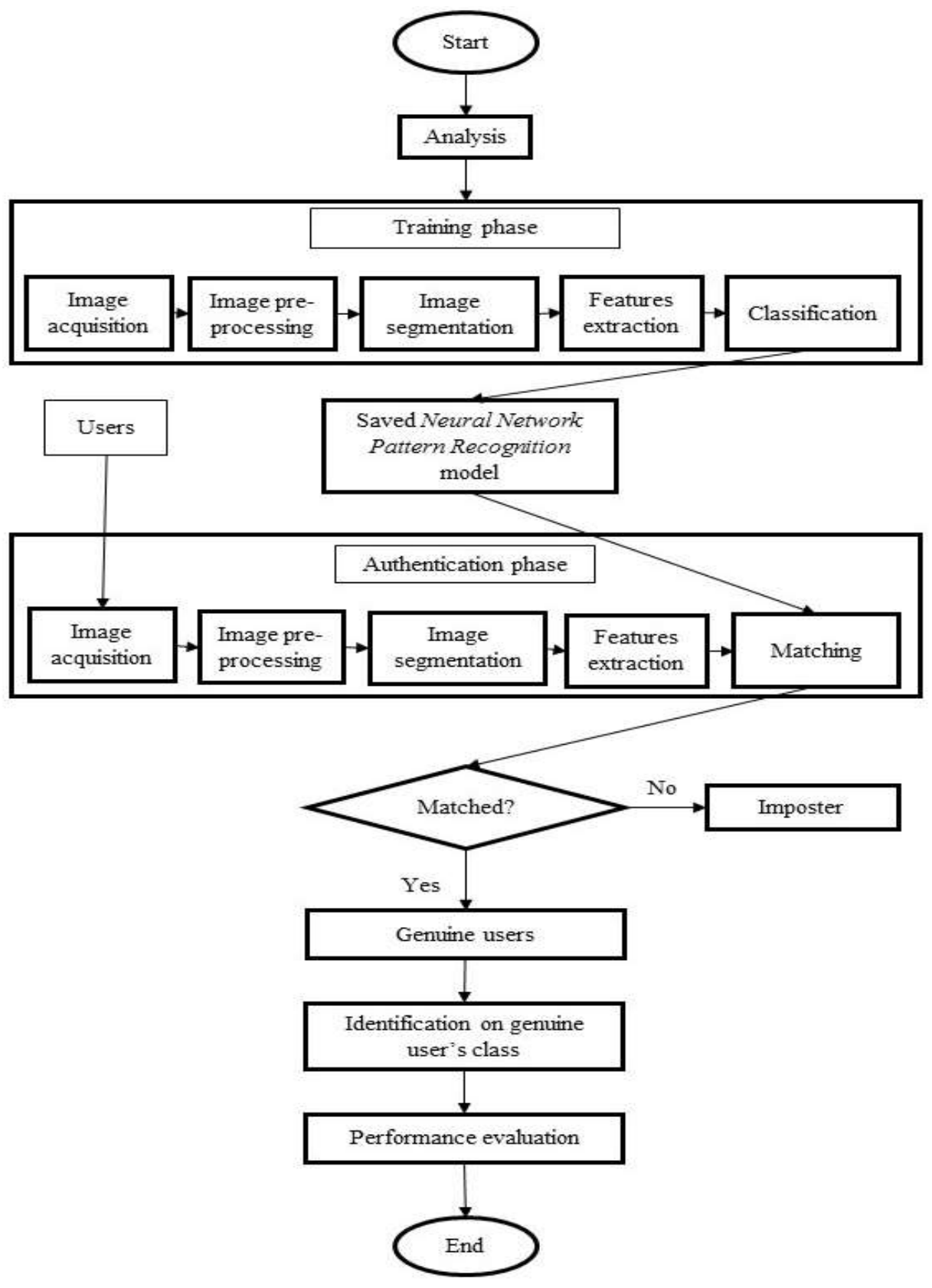

Figure 1. Flowchart of dorsal hand vein pattern authentication system using ANN

In the pre-processing stage, the images were cropped from $300 \times 240$ pixels into $60 \times 60$ pixels to obtain ROI. After cropping the images, mean filter, CLAHE enhancement and histogram equalization were applied to the cropped ROI to denoise, enhance and contrast the images respectively. The equalized image was then binarized to segment out the vein pattern. Feature extraction was done by extracting the LBP features which consists of 236 features from the binarized image. 
To classify the class of the hand vein images, ANN was created by using Neural Network Pattern Recognition Pattern App from Matlab 2019b as shown in Figure 2. The 2-layered feedforward NN consists of one input with 236 neurons, one hidden layer with 240 neurons, one output layer with 80 neurons and one output which consists of 80 neurons. The hidden layer contains weight, bias and tan-sigmoid transfer function, whereas the output layer has a softmax transfer function on top of weight and bias.

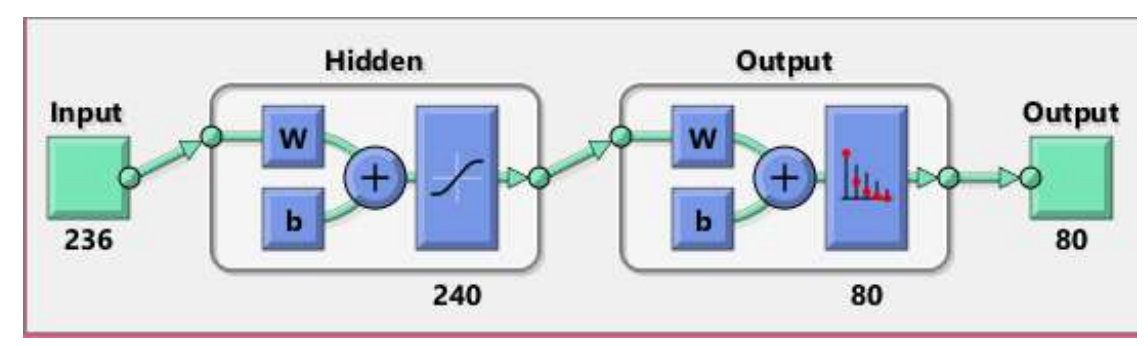

Figure 2. Structure of ANN

Each input neuron $X_{i}$ will be multiplied with its corresponding weight $W_{i}$ in the hidden layer. Summation of the product of each input neuron $X_{i}$ and its weight $W_{i}$ together with a bias $b$ is called a net, $n e t=\sum_{i=1}^{n} X_{i} W_{i}+b$

. The net will be inputted into the tan-sigmoid transfer function to give the continuous output between 0 and 1 .

$$
Y_{i}=f\left(\sum_{i=1}^{n} X_{i} W_{i}+b\right)
$$

The tan-sigmoid transfer function is given by $f(x)=\frac{1}{1+e^{-x}}$. The output from the hidden layer will be multiplied with the corresponding weight in the output layer. Summation of the product of output from the hidden layer and corresponding weight $W_{i}$ together with a bias $b$ in the output layer gives a new net. Later the new net will be passed to the softmax transfer function to give the final output, $Z_{i}=g\left(\sum_{i=1}^{n} Y_{i} W_{i}+b\right)$. softmax transfer function is a normalized exponential function given by

$$
g(y)_{i}=\frac{e^{y_{i}}}{\sum_{i=1}^{k} e^{y_{i}}}
$$

The input in this study is the LBP features (236 features $\times 240$ images) and the target data ( 80 classes $\times 240$ images) or equivalent to the dimension of 80 rows $x 240$ columns. Target was constructed according to the class of images where the binary value ' 1 ' denotes true class and the binary value ' 0 ' denotes false class. In other words, each column of target data contains ' 0 ' values except ' 1 ' at the corresponding class.

To train the network for classification, the 240 images were randomly divided into $90 \%$ training, $5 \%$ validation and $5 \%$ testing. Initially, the weight is randomly assigned, later the error between the predicted output and target will be calculated. The weight will be adjusted to minimize the errors using the scaled conjugate gradient (SCG) backpropagation algorithm [27] during the training process. The SGS algorithm combines both conjugate gradient (CG)and Levenberg-Marquardt algorithms to avoid time-consuming line search in $\mathrm{CG}$ algorithm.

The performance of the trained NN was measured using recognition rate, false rejection rate (FRR) and false acceptance rate (FAR) as below. In the training process, FR means a correct class was not identified, while FA indicates wrong class or for example, class 1 is identified as another class, etc. 


$$
\begin{aligned}
& \text { Recognition rate }=\frac{\text { number of recognizedi mages }}{\text { total number of images }} \times 100 \% \\
& F R R=\frac{\text { number of unrecognized images }}{\text { total numberof images }} \times 100 \% \\
& F A R=\frac{\text { number of misclass }}{\text { total number of images }} \times 100 \%
\end{aligned}
$$

The trained NN net was then saved to be used at the matching stage of the authentication phase. The workflow of the training phase can be represented in Figure 3. In the authentication phase, a graphic interface user (GUI) program was developed. 80 trained images of 80 users and 20 untrained images of 20 users which were directly selected from the Bosphorus Hand Vein Database were tested using this GUI. The images were sent to the image pre-processing to go through the mean filter, CLAHE enhancement and histogram equalization. Binarization was adopted to the pre-processed images. The LBP features of the binarized images were then extracted and matched with the trained NN net. The results of matching would be a genuine case or imposter case. For the genuine case, identification of the genuine user was done. The workflow of the authentication phase could be represented in Figure 4.

The performance analysis of the dorsal hand vein authentication will be calculated as given in the following Table 2. From Table 2, TA denotes true acceptance, FA denotes false acceptance, FR denotes false rejection and TR denotes true rejection. The meaning of TA, FA, FR and TR is given in Table 3.

To compare the performance of NN from Neural Net Pattern Recognition with other classifiers, machine learning algorithms from Classification Learner in App Matlab 2019 was used. In Classification Learner App, Input $(236$ x 240) and Target $(80$ x 240) in Neural Net Pattern Recognition App were supplied in a matrix only $(237 \times 240)$. In this case, the last row is Target which is not in binary number as before but labeled $1,2,3, \ldots, 80$ to indicate the class. There are decision tree (DT), Discriminant Analysis, Logistic Regression (LR), Naïve Bayes, support vector machine (SVM), Nearest Neighbor and ensemble classifiers in

\begin{tabular}{|c|c|c|c|}
\hline & Genuine user & Imposter & \\
\hline Acceptance(A) & TA & \multirow{3}{*}{ TR } & $\begin{array}{c}\text { Acceptance precision }= \\
T A\end{array}$ \\
\hline \multirow[t]{5}{*}{ Rejection (I) } & FR & & $\begin{array}{c}\overline{T A+F A} \\
\text { Rejection precision }= \\
T R\end{array}$ \\
\hline & & & $\overline{T R+F R}$ \\
\hline & Sensitivity=Recall & \multirow{3}{*}{ Specificity $=\frac{T R}{T R+F A}$} & \multirow{3}{*}{ Accuracy $=\frac{T A+T R}{T A+T R+F A+F R}$} \\
\hline & $T A$ & & \\
\hline & $\overline{T A+F R}$ & & \\
\hline
\end{tabular}
Classification Learner App.

Table 2. Confusion matrix

Table 3. The definition on the expressions

\begin{tabular}{ccc}
\hline Expression & Name & Definition \\
\hline TA & True acceptance & Genuine user with true class. \\
TR & True rejection & Imposter with no class. \\
FA & False acceptance & Genuine user with wrong class or imposter with wrong class. \\
FR & False rejection & Genuine user with no class. \\
\hline
\end{tabular}




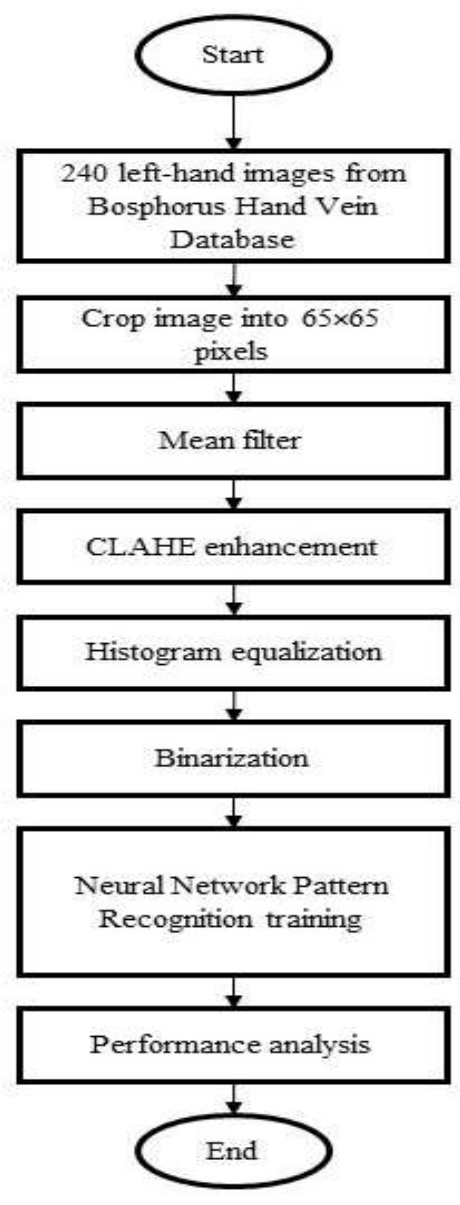

Figure 3. The workflow of the training phase of the dorsal hand vein authentication system using ANN

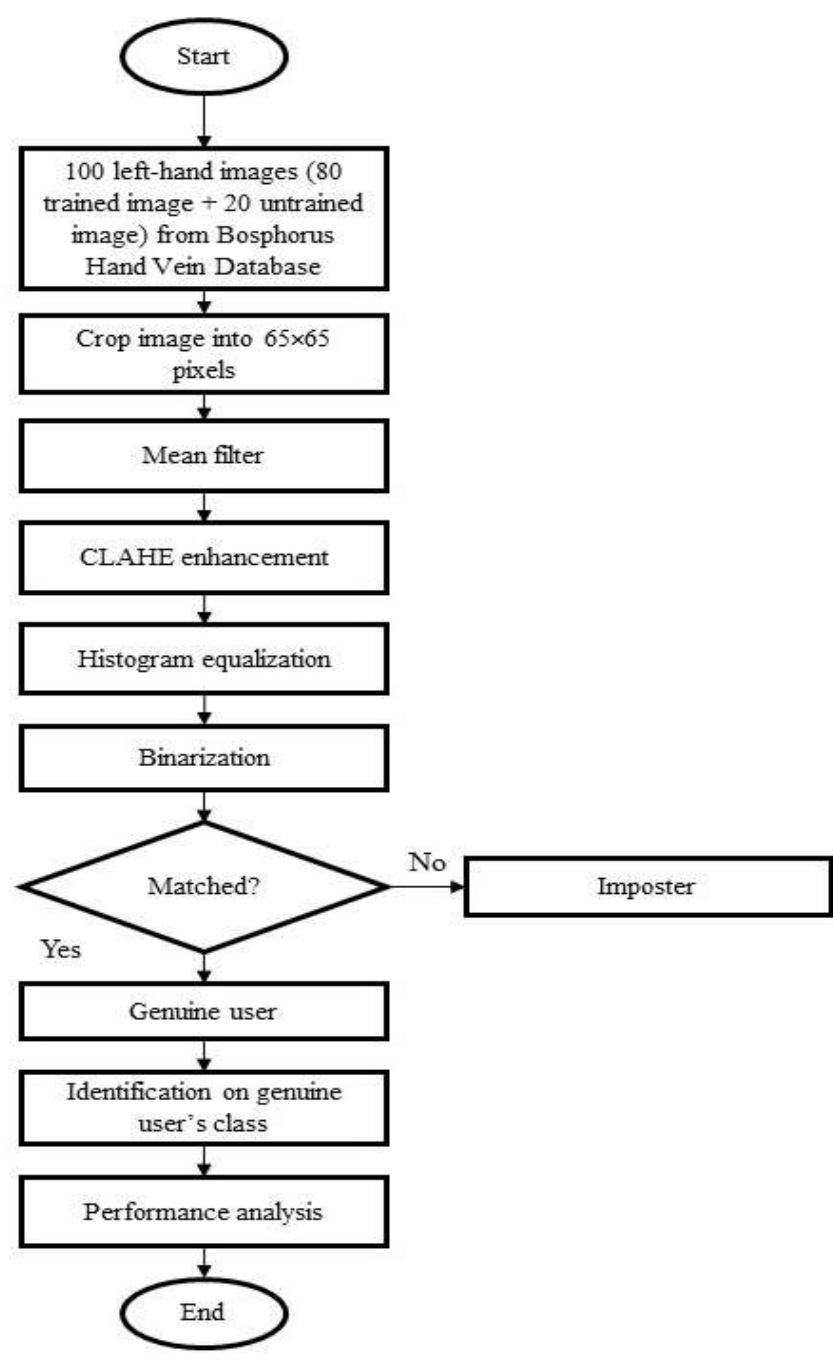

Figure 4. The workflow of the authentication phase in dorsal hand vein authentication system using ANN

\section{RESULTS AND DISCUSSION}

This section discusses the results during the training phase and the authentication phase.

\subsection{Training phase}

This subsection focuses on the performance analysis of the training phase. The confusion matrix for the 80 classes in the training phase is shown in Figure 5. Columns represent target (real) class, whereas rows indicate output (predicted) class from ANN. The main diagonal of the confusion matrix shows the recognized classes. Yellow color indicates all 3 training images at that particular class were correctly identified, green color denotes 1 image out of 3 images was recognized while blue color stands for 2 images per 3 images were correctly recognized. The total recognized images are 216 out of 240 , hence there is 24 images were unrecognized. From these 24 unrecognized classes, 3 are misclass as shown in red color at the off-diagonal in the confusion matrix. Thus, the performance parameters were calculated and tabulated as shown in Table 4 . The recognition rate of the dorsal hand vein using LBP features and ANN is 90\%. Figure 6 proves that the highest accuracy obtained using Classification Learner App is $20 \%$ from the KNN classifier. The significant difference of accuracy obtained in ANN and KNN may due to different labeling of target in both classifiers. On top of that, ANN allows retrain NN until the desired accuracy is obtained, but the Classification Learner App does not allow retrain. Besides, the accuracy results at each run in Classification Learner are different and not stable. Thus, Ann which yields a higher recognition rate was adopted to build the dorsal hand vein authentication system. 


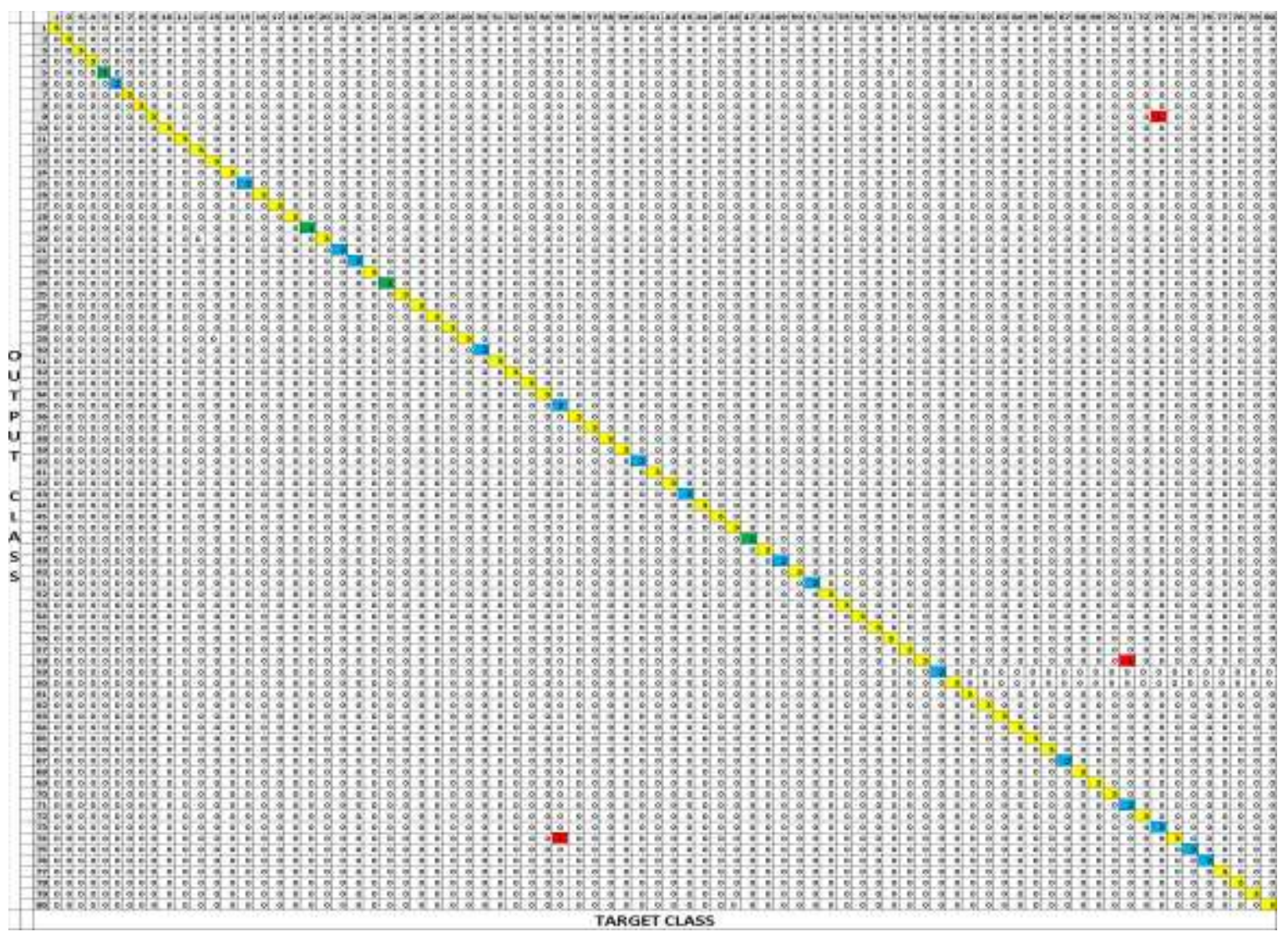

Figure 5. Confusion Matrix

Table 4. Performance analysis of the classification using ANN

\begin{tabular}{cc}
\hline Performance parameters & Percentage $(\%)$ \\
\hline Recognition rate & 90.00 \\
Precision & 98.63 \\
FRR & 10 \\
FAR & 1.25 \\
\hline
\end{tabular}

\begin{tabular}{|c|c|}
\hline $1.14 \quad$ KNJS & Accuracy $20.0 \%$ \\
\hline Last crange. Frie KNN & $238 \mathrm{23} 5$ featuras \\
\hline $1.15=\mathrm{KN} / \mathrm{N}$ & Accturace 6.79 \\
\hline Last change. Medium KNN & $236 \sqrt{236}$ fasturas \\
\hline $1.16 \quad$ IONN & Arcuracy $0.0 \%$ \\
\hline Last change: Coarse WWN & $236 \sqrt{236}$ fostur 98 \\
\hline $1.17 \quad 10 \mathrm{~N}$ & Accuracy $75 \%$ \\
\hline Last change: Cosine KNN & $206 \sqrt{236}$ features \\
\hline $1.18 \quad \mathrm{KON} \cdot \mathrm{I}$ & Accuracs. $79 \%$ \\
\hline Last crange: Cubic KNN & $220 / 236$ fealurgs \\
\hline 1.19 KNIN & Accurscy. 1639 \\
\hline
\end{tabular}

Figure 6. Accuracy of KNN

\subsection{Authentication phase}

This subsection discusses the developed dorsal hand vein pattern authentication system and the matching results. 100 images included 80 trained images and 20 untrained images were tested in the GUI program to validate the performance of the developed system. The GUI in Figure 7 and Figure 9 lets users select an image from the database. Once, each button of the Image Cropping, Mean Filter, CLAHE Enhancement, Histogram Equalization and Image Binarization buttons are selected respectively, the corresponding images are portrayed. Later, when, the Feature Extraction button is clicked, the LBP features will be extracted from the binarized image. Lastly, if the Matching button is pushed, the extracted LBP features will be matched with the imported NN net from the training phase. The result of the matching will be popped up as seen in Figures 8-9. Figure 7 displays the resultant images for a genuine user and its matching status is shown in Figure 8. The imposter case is displayed in Figure 9 if the developed system failed to verify the users. The performance of the result was tabulated in Table 5. From Table 5, the accuracy of the developed GUI program was $100 \%$, which proves that the system is successfully developed. 


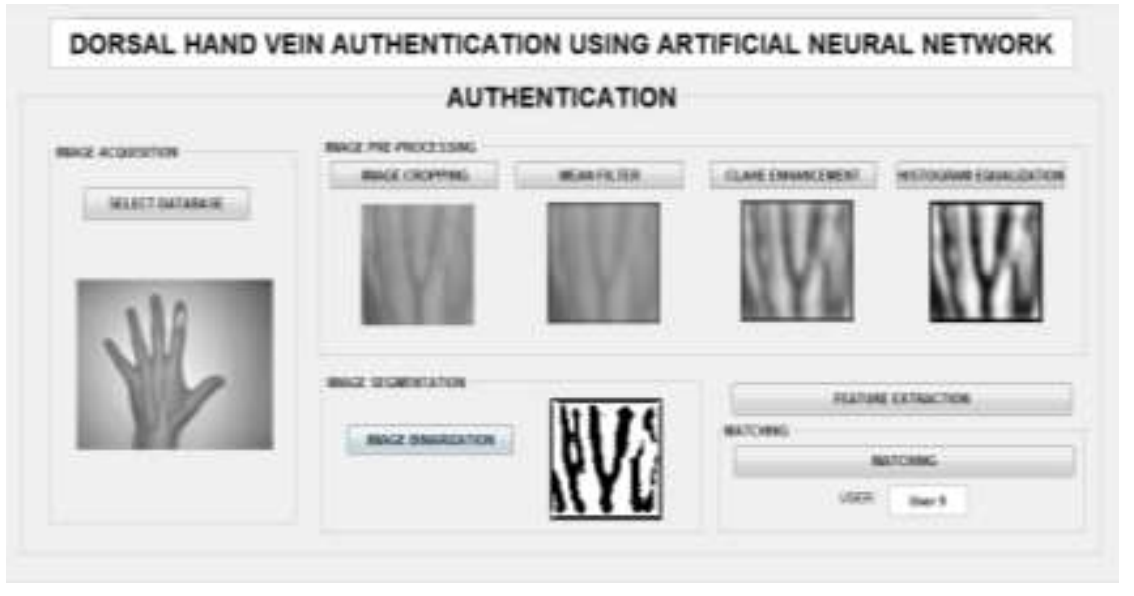

Figure 7. The resultant images with the identification of a genuine user

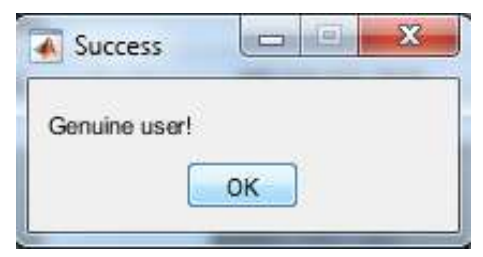

Figure 8. The message box of 'Genuine user!' pops up during the genuine case

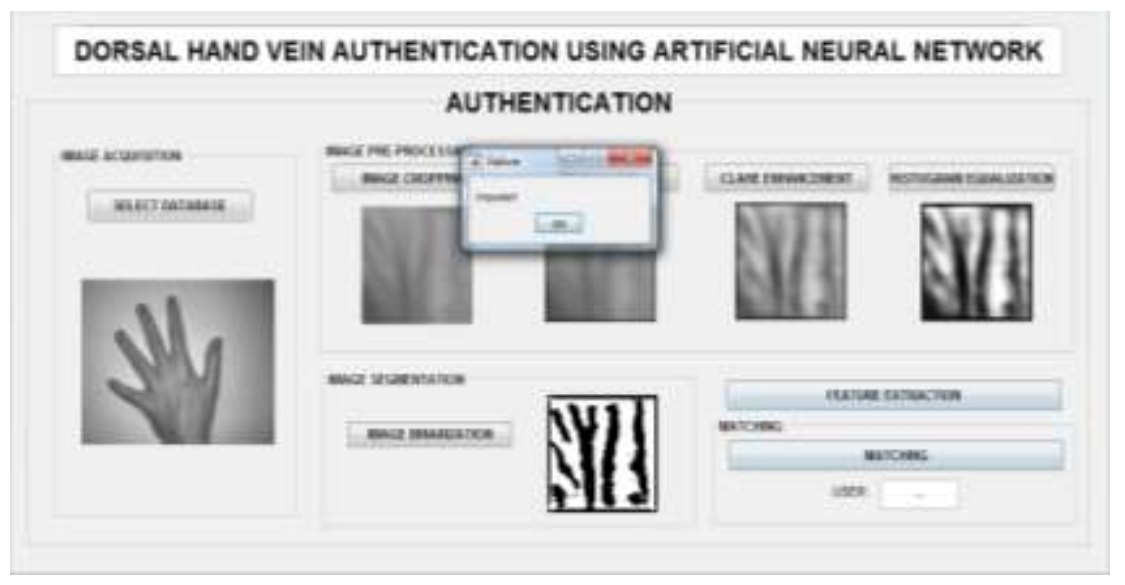

Figure 9. The resultant images with the identification of imposter user

\begin{tabular}{cc}
\multicolumn{3}{c}{ Table 5. Performance on the GUI program } \\
\hline Performance parameters & Percentage $(\%)$ \\
\hline Accuracy & 100.00 \\
Precision & 100.00 \\
Sensitivity & 100.00 \\
Specificity & 100.00 \\
\hline
\end{tabular}

\section{CONCLUSION}

In this work, dorsal vein pattern authentication was divided into two phases, which are the training phase and the authentication phase. To achieve the first objective, 240 training images from 80 users were acquired from the Bosphorus Hand Vein Database. Then, image processing methods were implemented to segment out the ROI followed by denoising, enhancement and binarization to obtain vein patterns. Later, the 
LBP features of the binarized dorsal vein pattern were gained. The LBP features were sent into the Neural Net Pattern Recognition App in Matlab 2019b to classify the vein pattern. The recognition rate, FRR and FAR using ANN model is $90 \%, 10 \%$ and $1.25 \%$. The trained ANN net model with LBP features was saved to the authentication phase. A GUI program was developed in the authentication phase with the aid of the NN net model to enable users to test their image. To evaluate the performance accuracy of the dorsal vein pattern authentication system, 100 images of 100 users were input and tested using the developed GUI. The accuracy of the testing and validation of the authentication system is $100 \%$. In a nutshell, this work shows a standard procedure of biometric authentication systems starts from image processing, image enhancement, segmentation, features extraction to ANN classification. The developed GUI system able to be modified to be used in real life as it is convenient and highly secure. Nevertheless, a practical biometric authentication systems process must be started with hardware development of the image acquisition process which is not covered in this study.

\section{ACKNOWLEDGEMENTS}

The authors would like to thank the Ministry of Higher Education Malaysia for financially supporting the work under the Fundamental Research Grant Scheme for Research Acculturation of Early Career Researchers (FRGS-RACER) scheme RACER/1/2019/ICT02/UTHM//1 and Research Fund E15501, Research Management Centre, UTHM.

\section{REFERENCES}

[1] M. Singaram, P. Preveena, J. Sabitha and M. Shalini, “ Dorsal Hand Vein Authentication System,” International Journal of Innovative Research in Science, Engineering and Technology, vol. 8, pp. 2566-2571, 2019.

[2] Y. Wang, K. Li and J. Cui, "Hand-dorsa Vein Recognition Based on Partition Local Binary Pattern," in International Conference on Signal Processing, ICSP 2010, IEEE, pp. 1671-1674, 2010.

[3] M. M. N. M. Heenaye and R. K. A. Subramanian, "Study of Dorsa Vein Pattern for Biometric Security," University of Mauritius Research Journal. 2009, vol. 15, pp. 17-25, 2009.

[4] Y. Wang, Y. Fan, W. Liao, K. Li, L. K. Shark and M. R. Varley, "Hand Vein Recognition Based on Multiple Keypoints Sets," $20125^{\text {th }}$ IAPR International Conference on Biometrics, IEEE,. pp. 367-371, 2012.

[5] K. Wang, Y. Zhang, Z. Yuan, and D. Zhuang, "Hand Vein Recognition Based on Multi Supplemental Features of Multi-Classifier Fusion Decision,"2006 IEEE International Conference on Mechatronics and Automation. IEEE, pp. 1790-1795, 2006.

[6] M. Rajalakshimi, V. Ganapathy, and R. Rengaraj, "Palm-Dorsal Vein Pattern Authentication Using Convolution Neural Network (CNN)," International Journal of Pure and Applied Mathematics, vol. 116, pp. 525-532, 2017.

[7] R. Raghavendra, J. Surbiryala, and C. Busch, "Hand Dorsal Vein Recognition: Sensor, Algorithms and Evaluation," 2015 IEEE International Conference on Imaging System and Techniques (IST), IEEE, pp. 1-6, 2015.

[8] L. Chen, H. Zheng, L. Li, P. Xie, and S. Liu, "Near-infrared Dorsal Hand Vein Image Segmentation by Local Thresholding Using Grayscale Morphology,"2007 $1^{\text {st }}$ International Conference on Bioinformatics and Biomedical Engineering, IEEE, pp. 868-871, 2007.

[9] P. Ramsoful and M. H. M. Khan, "Feature Extraction Technique for Dorsal Hand Vein Pattern," Third International Conference on Innovative Computing Technology (INTECH 2013), IEEE, pp. 49-53, 2013.

[10] A. Queslati, N. Feddaoui, and K. Hamrouni, "Identity Verification through Dorsal Hand Vein Texture Based on NSCT Coefficients," 2017 IEEE/ACS 14 th International Conference on Computer Systems and Application (AICCSA), IEEE, pp. 781-787, 2018.

[11] S. Bhosale, and M. R. Jadhav, "Dorsal Hand Vein Pattern Recognition System Based on Neural Network," 2017 International Conference of Electronics, Communication and Aerospace Technology (ICECA). IEEE, pp. 52-55, 2017.

[12] P. Gupta, and P. Gupta, "Multibiometric Authentication System Using Slap Fingerprints, Palm Dorsal Vein and Hand Geometry," IEEE Transactions on Industrial Electronics, vo1. 65, pp. 9777-9784, 2018.

[13] M. Shahin, A. Badawi, and M. Kamel. Biometric Authentication Using Fast Correlation of Near Infrared Hand Vein Patterns. International journal of biomedical sciences 2007. Vol. 2, no. 3, pp. 141-148, 2007.

[14] X. Li, D. Huang, R. Zhang, Y. Wang, and X. Xie, "Hand dorsal vein recognition by matching Width Skeleton Models," 2016 IEEE International Conference on Image Processing. (ICIP), IEEE, pp. 3146-3150, 2016.

[15] A. H. H. Alasadi and M.H. Dawood, "Dorsal hand-vein images recognition system based on grey level cooccurrence matrix and Tamura features," International Journal of Applied Pattern Recognition, vol. 4, pp. 207-226, 2017.

[16] B. Belean, M.Streza, S. Crisan, and S. Emerich, "Dorsal hand vein pattern analysis and Neural Network for biometric authentication system," Studies in Informatics and Control, vol. 26, pp. 305-314. 2017.

[17] Bosphorus Hand Database. DotNetNuke Corporation. [2010; 2019 October 2], available from http://bosphorus.ee.boun.edu.tr/hand/Home.aspx

[18] A. Kumar, and K.V. Prathyusha, "Personal authentication using hand vein triangulation and knuckle shape," IEEE Transactions on Image Processing, vol. 18, pp. 2127-2136, 2009. 
[19] S. Kulkarni, and M. Pandit, "Biometric Recognition System based on Dorsal Hand Veins," International Journal of Innovative Research in Science, Engineering and Technology, vol. 5, pp. 18899-18905, 2016.

[20] R. Malutan, S. Emerich, S. Crisan, O. Pop, and L. Lefkovits, "Dorsal hand vein recognition based on Riesz Wavelet Transform and Local Line Binary Pattern," 2017 3rd International Conference on Frontiers of Signal Processing (ICFSP), IEEE, pp. 146-150, 2017.

[21] Z. Honarpisheh and K. Faez, "An efficient dorsal hand vein recognition based on firefly algorithm," International Journal of Electrical and Computer Engineering (IJECE), vol. 3, pp. 30-41, 2013.

[22] S. Sanchit, M. Ramalho, P. L. Correia, and L. D. Soares, "Biometric identification through palm and dorsal hand patterns," 2011 IEEE EUROCON-International Conference on Computer as a Tool, IEEE, pp. 1-34, 2011.

[23] S. Zhao, Y. Wang, and Y. Wang, "Extracting Hand Vein Patterns from Low-Quality Images: A New Biometric Technique Using Low-Cost Devices," Fourth International Conference on Image and Graphics (ICIG 2007), IEEE, pp. 667-671, 2007.

[24] D. Sandhiya and B. Thiyaneswaran, "Extraction of dorsal palm basilic and cephalic hand vein features for human authentication system," 2017 International Conference on Wireless Communications, Signal Processing and Networking (WiSPNET), IEEE, pp. 2231-2235, 2018.

[25] M. U. Akram, H. M. Awan, and A. A. Khan, "Dorsal hand vein based person identification," 2014 4th International Conference on Image Processing Theory, Tools and Applications (IPTA), IEEE, pp. 1-6, 2015.

[26] Gopal, S. Srivastava, S. Bhardwaj, and S. Bhargava, "Fusion of palm-phalanges print with palmprint and dorsal hand vein," Applied Soft Computing Journal, vol. 47, pp. 12-20, 2016.

[27] M.F. Moller, “A Scaled Conjugate Gradient Algorithm for Fast Supervised Learning," Neural Networks, vol. 6, pp. 525-533, 1993.

\section{BIOGRAPHIES OF AUTHORS}

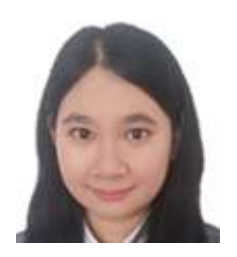

Sze Wei Chin obtained her Bachelor of Electronic Engineering major in Communication Engineering under the Faculty of Electrical and Electronic from Universiti Tun Hussein Onn Malaysia. Her research interests include image processing and machine learning. She is currently working as a test engineer in In Tech Electronic Sdn. Bhd.

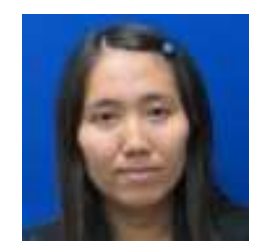

Kim Gaik Tay is an Associate Professor of Applied Mathematics under the Faculty of Electrical and Electronic Engineering at Universiti Tun Hussein Onn Malaysia. She has research interests in Nonlinear Waves, Optical Solitons, Fiber Optics Parametric Amplifier (FOPA), Numerical Methods, Forecasting, Breast Cancer Classification and Dorsal Hand Vein Pattern Recognition using machine learning techniques. She did her Bachelor, Master and Ph.D. in Applied Mathematics from the Universiti Teknologi Malaysia. She is a member of the International Association of Engineers (IAENG) and Persatuan Sains Matematik Malaysia (PERSAMA).

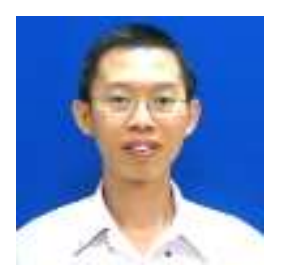

Chang Choon Chew is a senior lecturer in the Electronic Engineering Department of Universiti Tun Hussein Onn Malaysia. He obtained his B.Eng (Electrical) from Universiti Teknologi Malaysia, M.Eng (Electrical) from Kolej Universiti Teknologi Tun Hussein Onn and Ph.D. (Computer Engineering) from Okayama University. His research interest includes the Internet of Things, single-board computer, and WiFi communication.

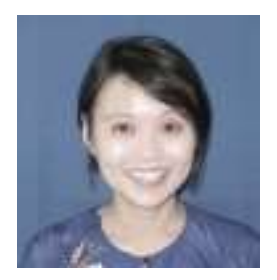

Audrey Huong is an Associate Professor in the Biomedical Engineering Modelling and Simulation Research Group under the Faculty of Electrical and Electronic Engineering at Universiti Tun Hussein Onn Malaysia. Her research interests are Biomedical Optics, Polarisation microscopy, Spectroscopy and Imaging. She completed her Bachelor from Kolej Universiti Teknologi Tun Hussein Onn, followed by a Ph.D. from the University of Nottingham, UK. She has a patent for a product named FACIOZ that has been developed under her supervision to test the oxygen level on skin.

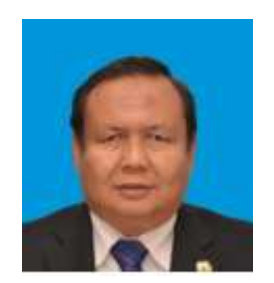

Ruzairi Abd Halim received a B. Eng. degree with Honours in Electronic System and Control Engineering in 1992 from Sheffield City Polytechnic, UK. He received his Ph.D. in Instrumentation \& Electronics Engineering from Sheffield Hallam University, UK in 1996. At present he is a Professor in the Engineering Faculty of Universiti Teknologi Malaysia. His current research interests are Process Tomography and Sensor Technology. 